\title{
Drift Mode Calculations in Nonaxisymmetric Geometry
}

\author{
G. Rewoldt, L.-P. Ku, and W. M. Tang \\ Plasma Physics Laboratory, Princeton University, \\ Princeton, New Jersey 08543-0451 \\ W. A. Cooper \\ Centre de Recherche en Physique des Plasmas, Ecole Polytechnique Fédérale de Lausanne, \\ PPB Ecublens, CH-1015 Lausanne, Switzerland
}

\begin{abstract}
A fully kinetic assessment of the stability properties of toroidal drift modes has been obtained for nonaxisymmetric (stellarator) geometry, in the electrostatic limit. This calculation is a comprehensive solution of the linearized gyrokinetic equation, using the lowest-order "ballooning representation" for high toroidal mode number instabilities, with a model collision operator. Results for toroidal drift waves destabilized by temperature gradients and/or trapped particle dynamics are presented, using three-dimensional magnetohydrodynamic equilibria generated as part of a design effort for a quasiaxisymmetric stellarator. Comparisons of these results with those obtained for typical tokamak cases indicate that the basic trends are similar.

$52.35 \mathrm{Qz}, 52.65 \mathrm{Tt}, 52.55 \mathrm{Hc}$
\end{abstract}

Typeset using REVTEX 


\section{INTRODUCTION}

Major interest in plasma confinement utilizing nonaxisymmetric toroidal configurations has been very evident in the international fusion research community. These include, for example, the Large Helical Device (LHD) in Japan, which has just started operation, ${ }^{1}$ the Wendelstein-7X (W7X) device under construction in Germany, ${ }^{2}$ and the exploration of tokamak-stellarator hybrid configurations, such as the National Compact Stellarator Exper-

iment (NCSX), currently being pursued in the United States. ${ }^{3}$ Accordingly, there is strong incentive to gain a better understanding of the associated anomalous transport properties. This has motivated the present investigation of the underlying linear microinstabilities.

The approach taken is to begin with the well-established axisymmetric linear eigenvalue microinstability code, FULL, ${ }^{4,5}$ which has been successfully and extensively applied to tokamak plasmas. This code has now been systematically extended to enable the investigation of the more general case of nonaxisymmetric stellarator geometry. Thus, all of the kinetic dynamics previously included in the axisymmetric version are retained in the nonaxisymmetric version. In particular, it includes trapped particles, finite Larmor radius effects to all orders, banana orbital dynamics, bounce frequency and transit frequency and magnetic drift frequency resonances, equilibrium shaping effects, and so on, for all species. Any number of plasma species can be included. This high-n (toroidal mode number), ballooning representation code now makes use of three-dimensional magnetohydrodynamic (MHD) equilibria from the VMEC code, ${ }^{6,7}$ which works in VMEC coordinates. The MHD equilibrium is transformed to Boozer coordinates ${ }^{8}$ by the TERPSICHORE code, ${ }^{9}$ and needed data along a chosen magnetic field line for the kinetic instability calculation in FULL is constructed by the VVBAL code. ${ }^{10}$ The extended FULL code finds all of the trapped-particle turning points along the field line numerically, so that all of the toroidally-trapped and helically-trapped particles are included automatically in the calculation. The Krook model collision operator previously used for axisymmetric geometry has also been extended for multiple classes of trapped particles. The needed changes have now been made in the electrostatic version of 
the FULL code.

The extended code is validated by comparing results from the previous axisymmetric version of the FULL code, using an axisymmetric MHD equilibrium ${ }^{11}$ generated by the JSOLVER ${ }^{12}$ code in PEST-I coordinates, ${ }^{13}$ with results calculated by the new nonaxisymmetric version of the FULL code, using a "matched" three-dimensional MHD equilibrium calculated by the VMEC code, with all nonaxisymmetric components equal to zero. Results are also presented for electrostatic toroidal drift modes (trapped-electron modes, ion temperature gradient modes) for an MHD equilibrium generated as part of the ongoing NCSX stellarator design effort. ${ }^{3}$ Various instability parameters are varied and the resulting effects on the instability linear growth rates and real frequencies are evaluated. In general, the results are qualitatively similar to those obtained for typical tokamak cases.

The necessary changes in the FULL code for nonaxisymmetric MHD equilibria relative to the previous axisymmetric code are described in Sec. II. Results for several collisionless cases are presented and compared in Sec. III. The extended model collision operator and collisional results are described in Sec. IV. Conclusions are given in Sec. V.

\section{CHANGES IN EQUATIONS}

For the three-dimensional VMEC MHD equilibrium, after the transformation to Boozer coordinates, the spatial coordinates are the flux surface label $s$, the poloidal angle $\theta$, and the toroidal angle $\zeta$. A more detailed description of this coordinate system and of the corresponding ballooning representation calculation for ideal MHD ballooning modes is given in Ref. 10. In the present lowest-order ballooning representation, the coordinate $\theta$ acts as a nonperiodic coordinate along the equilibrium magnetic field line. Defining $B(\theta) \equiv|\mathbf{B}(\theta)|$ and the unit vector $\mathbf{b} \equiv \mathbf{B}(\theta) / B(\theta)$ along the equilibrium magnetic field line, the quantities from the MHD equilibrium calculation which are needed for the the kinetic microinstability

calculation are $B(\theta), \mathbf{b} \cdot \nabla, k_{\perp}^{2}(\theta)$, the magnetic curvature drift $\mathbf{k}_{\perp} \cdot\{\mathbf{b} \times[(\mathbf{b} \cdot \nabla) \mathbf{b}]\}$, and the magnetic gradient drift $\mathbf{k}_{\perp} \cdot(\mathbf{b} \times \nabla B)$, all evaluated in the lowest-order ballooning 
representation. Using results from Ref. 10, we obtain

$$
\begin{gathered}
\mathbf{b} \cdot \nabla=\frac{\Psi^{\prime}(s)}{\sqrt{g} B} \frac{\partial}{\partial \theta}, \\
k_{\perp}^{2}(\theta)=n^{2} \frac{\sqrt{g} B^{2}}{\left[\Psi^{\prime}(s)\right]^{2}}\left[C_{p}+C_{s}\left(\theta-\theta_{0}\right)+C_{q}\left(\theta-\theta_{0}\right)^{2}\right], \\
\mathbf{k}_{\perp} \cdot(\mathbf{b} \times \nabla B)=B \mathbf{k}_{\perp} \cdot\{\mathbf{b} \times[(\mathbf{b} \cdot \nabla) \mathbf{b}]\}+\frac{n p^{\prime}(s)}{\Psi^{\prime}(s)},
\end{gathered}
$$

and

$$
\mathbf{k}_{\perp} \cdot\{\mathbf{b} \times[(\mathbf{b} \cdot \nabla) \mathbf{b}]\}=n \frac{B \Psi^{\prime}(s)}{2 p^{\prime}(s) \sqrt{g}}\left[d_{p}+d_{s}\left(\theta-\theta_{0}\right)\right] .
$$

Here, $2 \pi \Psi(s)$ is the poloidal magnetic flux, $p(s)$ is the pressure, $g$ is the determinate of the metric tensor, and $\sqrt{g}$ is the Jacobian of the Boozer magnetic coordinate system. The prime $(')$ indicates a derivative with respect to $s$, and $\theta_{0}$ (also called $\theta_{k}$ ) is the ballooning parameter. ${ }^{10}$ Algebraic expressions for $C_{p}, C_{s}, C_{q}, d_{p}$, and $d_{s}$ are given in Ref. 10. All of these quantities are obtained as part of the MHD equilibrium solution and its mapping to Boozer coordinates.

The solution of the linearized gyrokinetic equation given in Ref. 4 can be used, with substitutions for $\mathbf{b} \cdot \nabla, k_{\perp}^{2}(\theta)$, and the magnetic drift frequency $\omega_{d j}(\theta)=\left(v_{\|}^{2} / \Omega_{j}\right) \mathbf{k}_{\perp} \cdot\{\mathbf{b} \times$ $[(\mathbf{b} \cdot \nabla) \mathbf{b}]\}+\left(v_{\perp}^{2} / 2 \Omega_{j} B\right) \mathbf{k}_{\perp} \cdot(\mathbf{b} \times \nabla B)$ using the previous expressions, where $\Omega_{j} \equiv e_{j} B / m_{j} c$. In the electrostatic limit, $\hat{A}_{\|}$and $\hat{A}_{\perp}$ are set to zero in the expressions in Ref. 4 , and only the quasineutrality condition is used, but the pressure correction term in Eq. 3 here is retained. The resulting integral equation along the magnetic field is converted to a matrix equation by expanding the unknown perturbed electrostatic potential in terms of chosen basis functions, and the matrix equation is solved by standard methods, as described in detail in Ref. 4 . 


\section{COLLISIONLESS RESULTS}

\section{A. Axisymmetric comparison}

For the purpose of validating the extended nonaxisymmetric version on the FULL code, we compare results from this version with those from the earlier axisymmetric version, which in turn has been compared with an initial value code embodying essentially the same physics, ${ }^{14}$ and with a gyrofluid code embodying a good approximation to the same physics. ${ }^{15}$ For this purpose we use an axisymmetric, two dimensional, fixed boundary MHD equilibrium described in Ref. 11 for the ARIES-RS reactor design with aspect ratio 3.0, calculated using the JSOLVER code, and a "matched" MHD equilibrium computed with the three dimensional VMEC code, for which all of the nonaxisymmetric components of $\mathbf{B}$ are zero. This equilibrium is computed with low $\beta\left(\beta_{N}=0.1\right.$ with $\left.B_{0}=6.917 \mathrm{~T}\right)$ and we choose $\eta_{i}=\eta_{e}=3.0$ and $T_{i}=T_{e}=15.74 \mathrm{keV}$ on the chosen magnetic surface (which is surface 36 of 48), including only electrons and background deuterium ions in the kinetic calculation. For the collisionless electrostatic drift mode, with ballooning parameter $\theta_{0}=0$, results for the linear growth rate $\gamma$ are shown in Fig. 1(a) and for the real frequency $\omega_{r}$ in Fig. 1(b) versus $k_{\perp} \rho_{i}(\theta=0)(\propto n)$. It can be seen that there is good agreement for both $\gamma$ and $\omega_{r}$ over the range of $k_{\perp} \rho_{i}(\theta=0)$ from zero to one. The behavior seen here for the eigenfrequency of the toroidal drift mode is typical, with the growth rate having a maximum for $k_{\perp} \rho_{i} \simeq 0.4$, and with the real frequency making a transition from the electron diamagnetic direction to the ion diamagnetic direction as $k_{\perp} \rho_{i}(\theta=0)$ increases.

\section{B. NCSX cases}

We now make use of a stellarator MHD equilibrium generated in the course of the ongoing design study for NCSX, ${ }^{3}$ which is quasiaxisymmetric with three field periods (QAS3), which

means that the equilibrium magnetic field strength is approximately symmetric in $\zeta$ after the transformation to Boozer coordinates. The particular equilibrium used is called QAS3- 

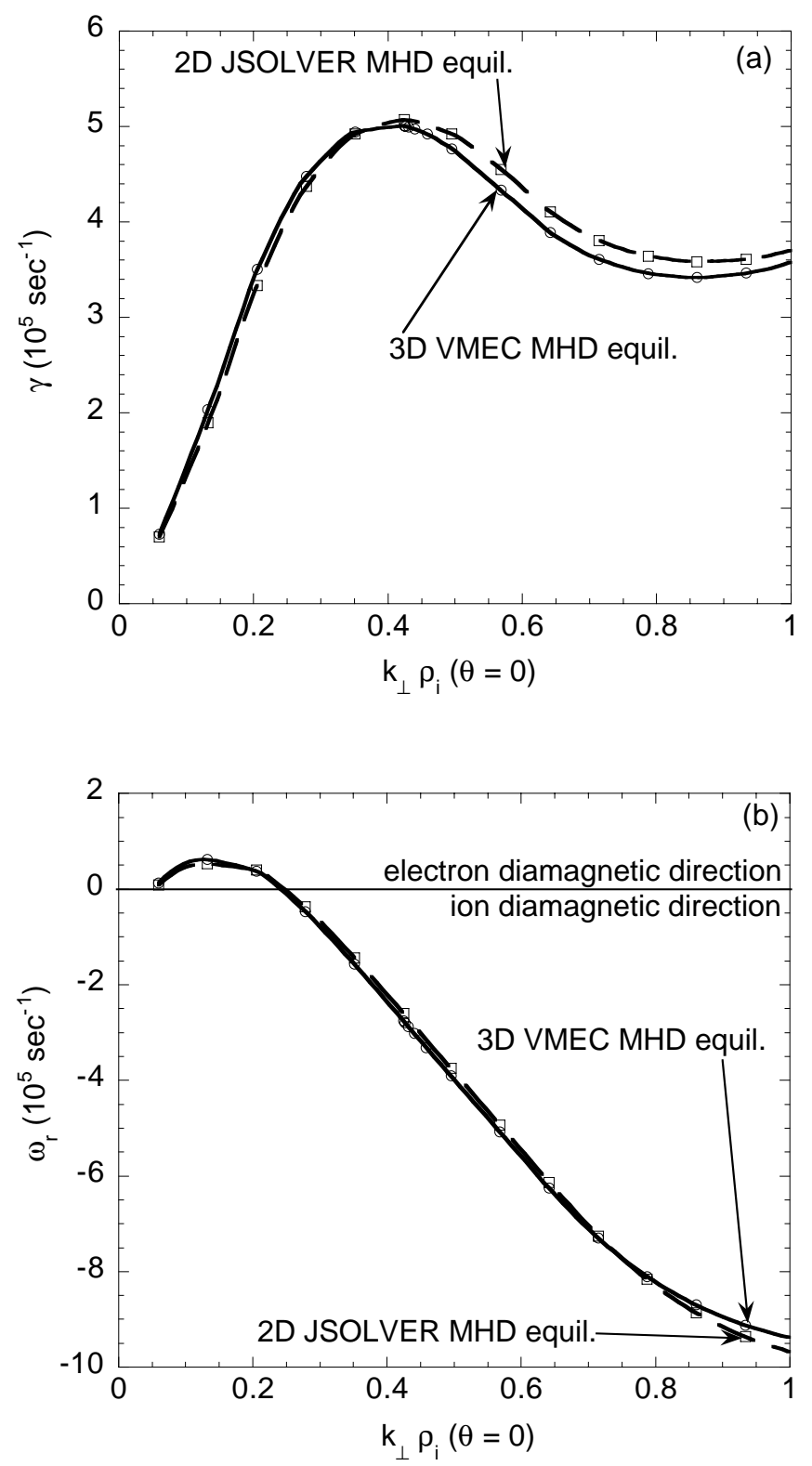

FIG. 1. Electrostatic, collisionless toroidal drift mode results for (a) growth rate $\gamma$ and (b) real frequency $\omega_{r}$ for axisymmetric ARIES-RS case with aspect ratio 3.0 and $\beta_{N}=0.1$. Here, $\eta_{i}=\eta_{e}=3.0, T_{e}=T_{i}=15.74 \mathrm{keV}$, and $B_{0}=6.917 \mathrm{~T}$. 
$\mathrm{C} 82,{ }^{16}$ here calculated in fixed boundary mode with very low volume average $\beta,\langle\beta\rangle=$ $0.01 \%$. As a standard set of local parameters for the microinstability calculation, we choose magnetic surface 88 of $100\left(s=0.875 \simeq\right.$ average $(\mathrm{r} / \mathrm{a})^{2}$, average $\left.r / a \simeq 0.935\right)$, field line label $\alpha \equiv \zeta-q \theta=\pi / 3=1.0472$, ballooning parameter $\theta_{0}=0$, toroidal mode number $n=25\left(k_{\perp} \rho_{i}(\theta=0)=0.3983\right), T_{i}=T_{e}=1.0 \mathrm{keV}$, and $\eta_{i}=\eta_{e}=3.0$. These parameters will later be varied one by one to investigate the effects on the linear growth rates and real frequencies. We retain only electrons and background deuterium ions in the calculation.

The input functions along the equilibrium magnetic field line constructed by the VVBAL code and input by the FULL code for this case extend over $252 \pi$ field periods in the (nonperiodic) ballooning coordinate $\theta$, from $-12.5 \times 2 \pi$ to $12.5 \times 2 \pi$. The magnitude of the equilibrium magnetic field $B(\theta)$ is shown in Fig. 2 for the center period, $-\pi<\theta<\pi$. A given pitch angle corresponds to a constant level on this kind of plot, and the intersections with the $B$ curve show the locations of the trapped particle turning points. As an example, for the 1.10 level line in Fig. 2, there are only two turning points in this $2 \pi$ period, and this corresponds to a single class of toroidally trapped particles, the class which is familiar from axisymmetric tokamak geometry. However, for the 1.33 level line, there are 12 turning points in this $2 \pi$ period, corresponding to 7 classes of trapped particles with one or both turning points in this period. The other 6 classes, in addition to the class of toroidally trapped particles whose two turning points surround $\theta=0$, are considered to be helically trapped particles. For the 0.94 level line, there are four turning points in this period, corresponding to two classes of trapped particles, which could be considered to be hybrid classes of trapped particles. However, this kind of designation makes no difference to the actual numerical calculation. All of the turning points and trapped particle classes are found numerically by the FULL code for each pitch angle.

The corresponding curve for $k_{\perp}^{2}(\theta)$ is shown in Fig. 3 and for the curvature drift $\mathbf{k}_{\perp} \cdot\{\mathbf{b} \times[(\mathbf{b} \cdot \nabla) \mathbf{b}]\}$ in Fig. 4. Positive curvature drifts are "bad" or destabilizing and negative curvature drifts are "good" or stabilizing. The linear eigenfunction for the perturbed electrostatic potential $\hat{\phi}(\theta)$ for the collisionless, electrostatic toroidal drift mode is 


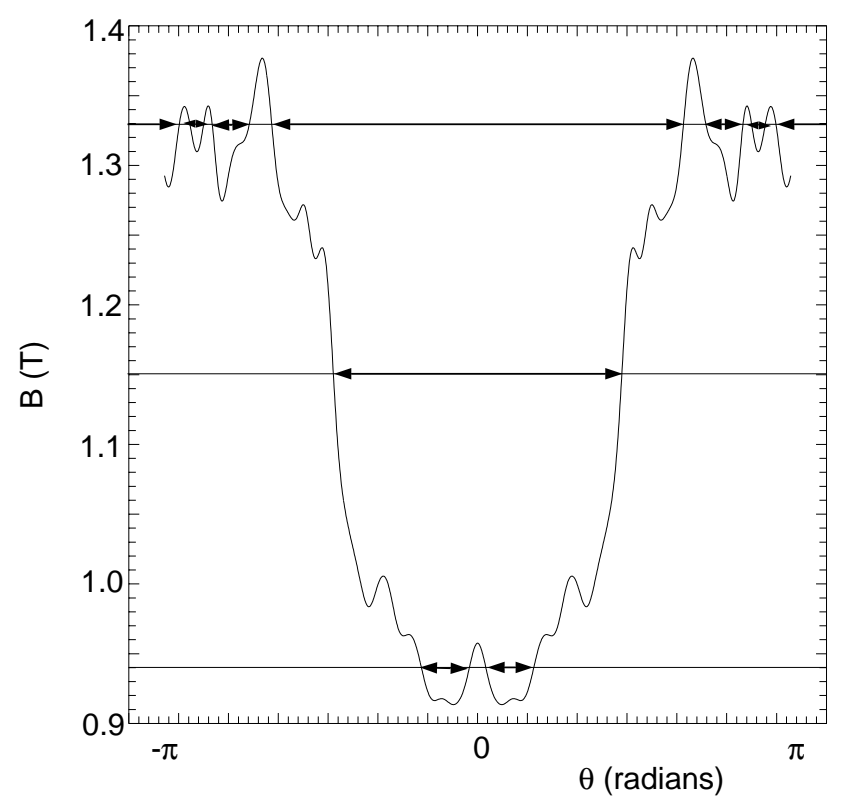

FIG. 2. Variation of $B(\theta)$ along chosen magnetic field line with $\alpha=1.0472, \theta_{0}=0$, on magnetic surface 88 of $100(s=0.875)$, for NCSX design case QAS3-C82 (fixed boundary), with $\langle\beta\rangle=0.01 \%$.

shown in Fig. 5, for the eigenmode with the smallest number of nodes along the field line, which normally has the highest linear growth rate. The eigenfunction is well localized in the toroidal magnetic field well around $\theta=0$ in Fig. 2, and responds only weakly to the helical magnetic field wells at larger values of $|\theta|$, for this case.

The results of varying $\eta_{i}=\eta_{e}$ are shown for $\gamma$ in Fig. 6(a) and for $\omega_{r}$ in Fig. 6(b). In this process, the total pressure gradient is kept fixed, consistent with the MHD equilibrium, so that the density gradient decreases and the electron and ion temperature gradients increase, as $\eta_{i}=\eta_{e}$ increases. For this case, in the collisionless limit, there are actually two distinct roots. There is the one we have already referred to for $\eta_{i}=\eta_{e}=3.0$, which we label as the ion temperature gradient mode (ITG) root, in correspondence with the usual nomenclature for tokamak modes, which is unstable for all $\eta_{i}=\eta_{e}$ above a critical value 0.65 . The calculation for this root still includes trapped particles, which are destabilizing. There is another root, which we label as the trapped-electron mode (TEM) root, again in correspondence with tokamak nomenclature, which is unstable for all $\eta_{i}=\eta_{e}$ less than a critical value, 1.06. 


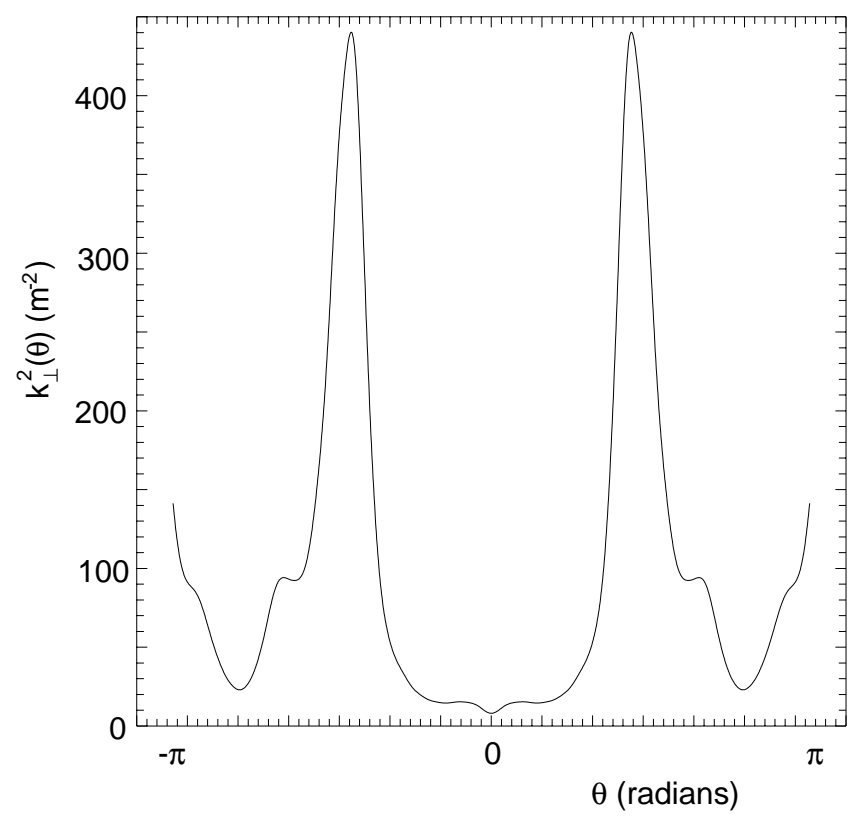

FIG. 3. Variation of $k_{\perp}^{2}(\theta)$ along chosen magnetic field line, for case of Fig. 2.

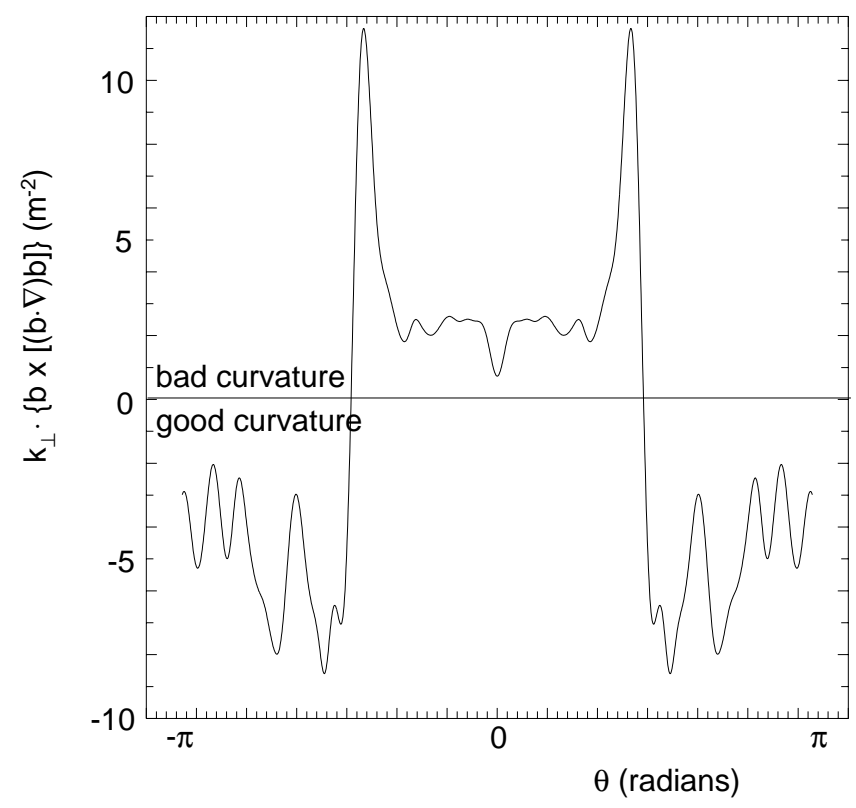

FIG. 4. Variation of curvature drift $\mathbf{k} \cdot\{\mathbf{b} \times[(\mathbf{b} \cdot \nabla) \mathbf{b}]\}$ along chosen magnetic field line, for case of Fig. 2. 


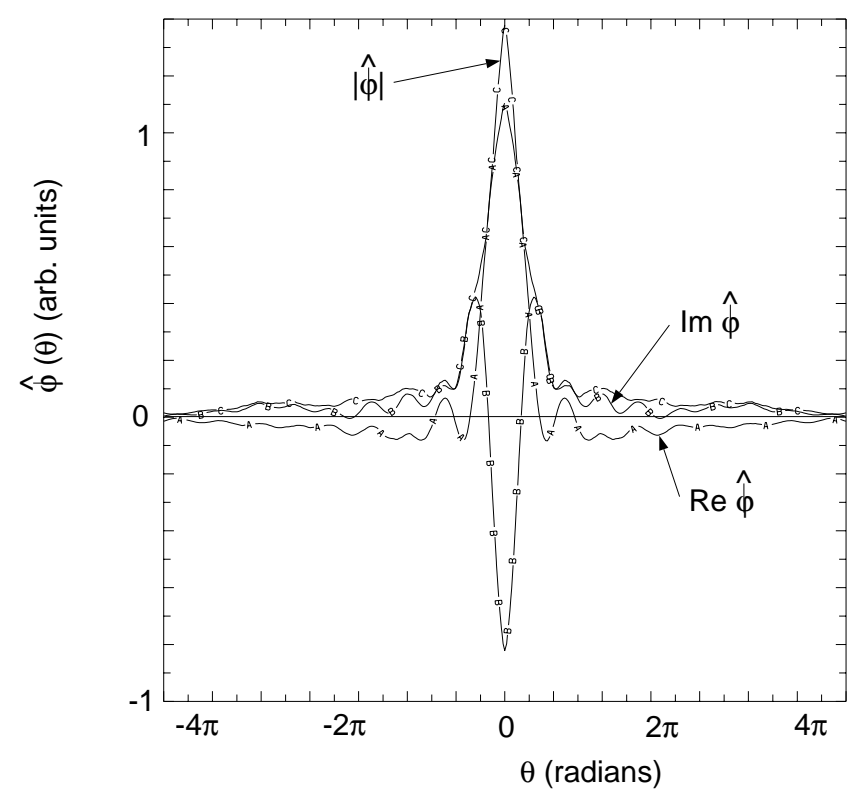

FIG. 5. Eigenfunction of electrostatic, collisionless toroidal drift mode (ITG root) along chosen magnetic field line, for case of Fig. 2 with electrons and deuterium ions only, with $\eta_{i}=\eta_{e}=3.0$, $T_{i}=T_{e}=1.0 \mathrm{keV}$, and $n=25\left(k_{\theta} \rho_{i}(\theta=0)=0.3983\right)$.

In many tokamak cases, ${ }^{17}$ these two roots will "hybridize" together, to form a single root which is continuously unstable for all positive $\eta_{i}$, sometimes referred to as the "trappedelectron- $\eta_{i}$ mode" (though they sometimes also remain as separate roots), but that did not happen for this case, because the two real frequencies are too widely separated. For the TEM root, the real frequency is always in the electron diamagnetic direction. For the ITG root, the mode starts out in the electron diamagnetic direction for small $\eta_{i}=\eta_{e}$, but makes a transition to the ion diamagnetic direction as $\eta_{i}=\eta_{e}$ increases. Here, we took $n=25$ $\left(k_{\perp} \rho_{i}(\theta=0)=0.3983\right)$ for the ITG root and $n=27\left(k_{\perp} \rho_{i}(\theta=0)=0.4302\right)$ for the TEM root. The effects of collisions will be discussed in Sec. IV.

The variation of $\gamma$ and $\omega_{r}$ with $n$ or $k_{\perp} \rho_{i}(\theta=0) \propto n$ is shown in Fig. 7 for the ITG root (with $\eta_{i}=\eta_{e}=3.0$ ) and for the TEM root (with $\eta_{i}=\eta_{e}=0.0$ ). The real frequency of the TEM root is always in the electron diamagnetic direction, for this range of $n$, while that for the ITG root starts out in the elctron diamagnetic direction at small $n$, but makes a transition to the ion diamagnetic direction as $n$ increases. This same behaviour is seen in 

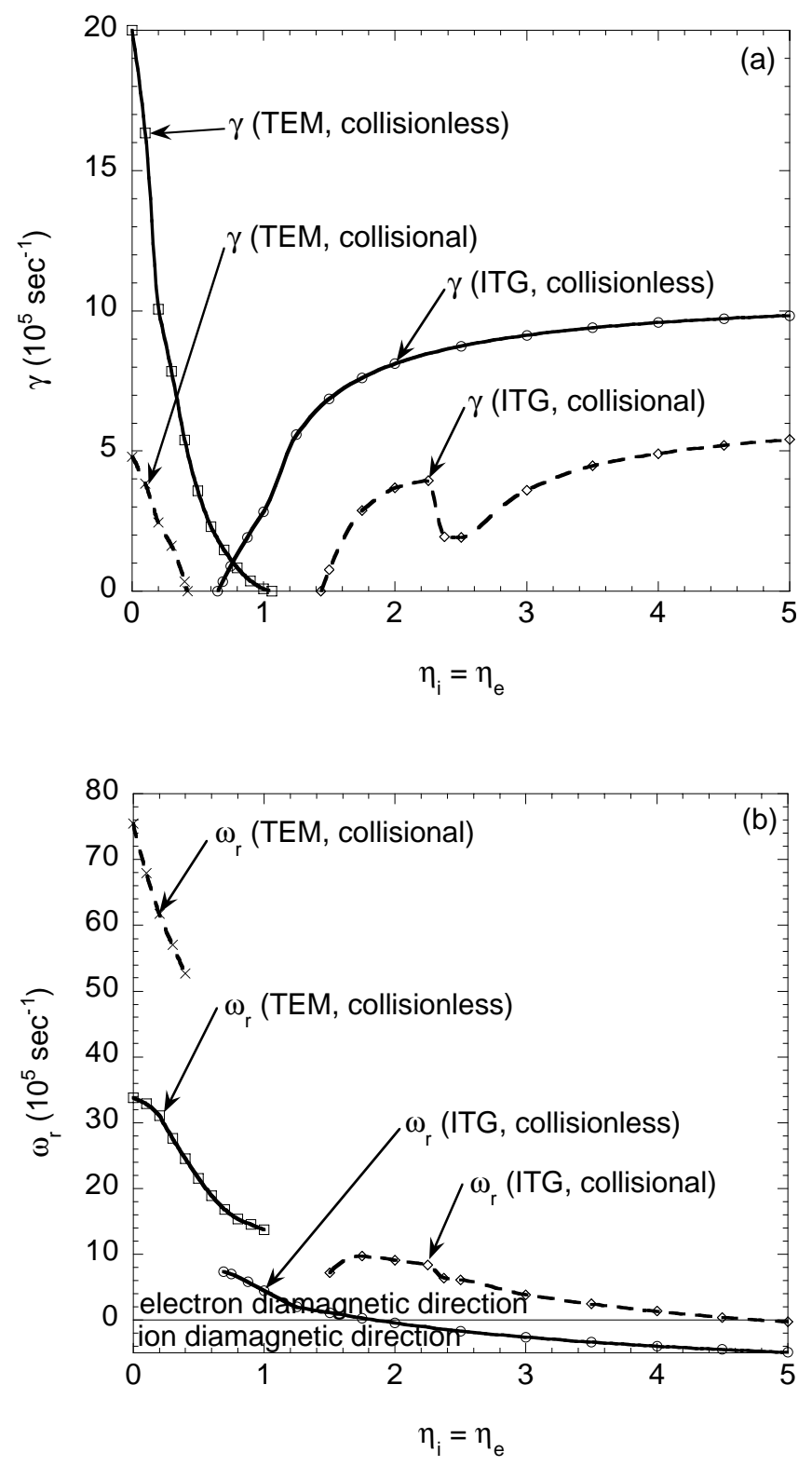

FIG. 6. Variation of (a) linear growth rate $\gamma$ and (b) real frequency $\omega_{r}$ with $\eta_{i}=\eta_{e}$ for ITG and TEM roots, for case of Figs. 2 and 5. Here, $n=25\left(k_{\theta} \rho_{i}(\theta=0)=0.3983\right)$ for the ITG root and $n=27\left(k_{\theta} \rho_{i}(\theta=0)=0.4302\right)$ for the TEM root, with $n_{e}=2.828 \times 10^{20} \mathrm{~m}^{-3}\left(\nu_{e}^{*}=0.7665\right)$ for the collisional cases. 
typical tokamak cases. The growth rates for both roots have maxima for $k_{\perp} \rho_{i}(\theta=0) \simeq 0.4$ to 0.5 , which is again typical for tokamak results also.

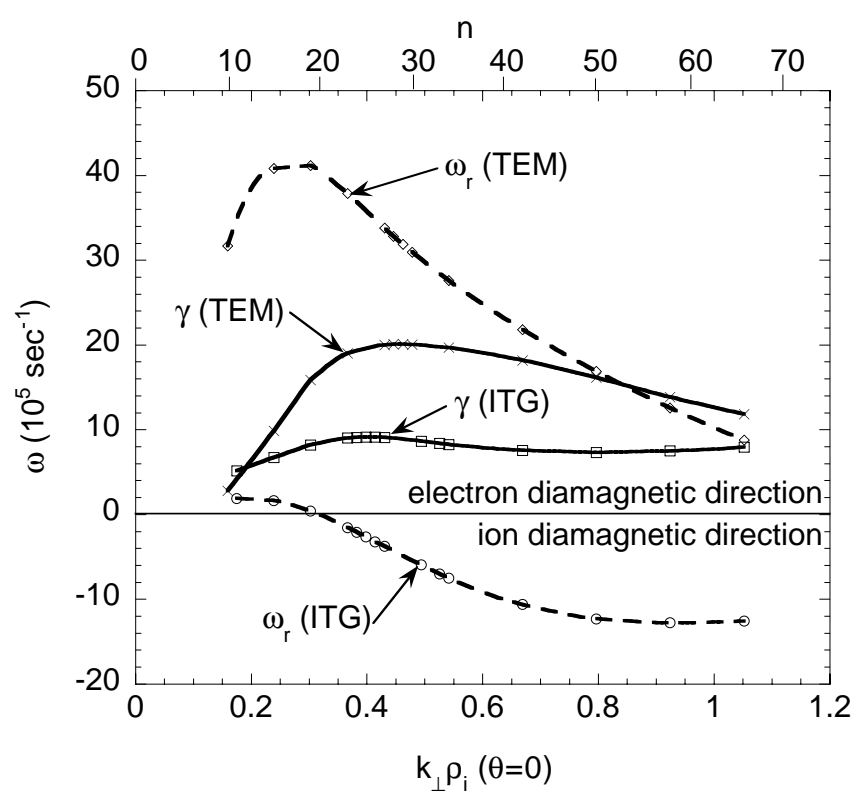

FIG. 7. Variation of $\gamma$ and $\omega_{r}$ with $n$ or $k_{\theta} \rho_{i}(\theta=0)$, for case of Figs. 2 and 5 with $\eta_{i}=\eta_{e}=3.0$ for ITG root and $\eta_{i}=\eta_{e}=0.0$ for TEM root.

The variation of the eigenfrequency for the ITG root with the ballooning parameter $\theta_{0}$ (also called $\theta_{k}$ ) is shown in Fig. 8. The variation is symmetric around $\theta_{0}=0$, and the growth rate is a maximum there. This behaviour is again typical of tokamak cases also. The effect of varying $\alpha \equiv \zeta-q \theta$ is shown in Fig. 9. The value of $\alpha$ determines the particular magnetic field line considered on the chosen magnetic surface. In axisymmetric geometry, this is an ignorable coordinate. In the present three-period equilibrium, $\omega$ is periodic in $\alpha$ with period $2 \pi / 3$, and the growth rate is seen to have a maximum for $\alpha=\pi / 3=1.0472$, i.e., half way through one period, and is symmetric around that maximum.

The variation of $\gamma$ and $\omega_{r}$ with the temperature ratio $T_{e} / T_{i}$ is shown in Fig. 10 for the ITG root (including trapped-particle effects). Here, two different situations are considered. In one, $T_{e}$ is varied while $T_{i}$ is kept fixed. In the other, $T_{i}$ is varied while $T_{e}$ and $k_{\perp} \rho_{i}(\theta=0)$ are kept fixed, with $n$ also being varied so as to compensate for the changing $T_{i}$. It is 


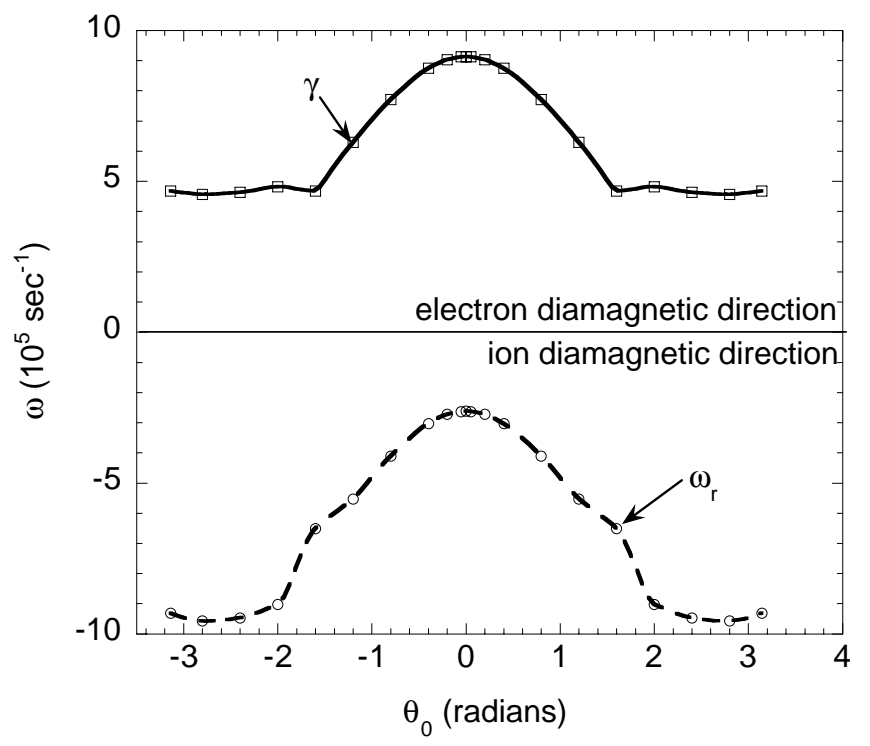

FIG. 8. Variation of $\gamma$ and $\omega_{r}$ with ballooning parameter $\theta_{0}$, for ITG root with $\eta_{i}=\eta_{e}=3.0$ and $n=25$, for case of Figs. 2 and 5.

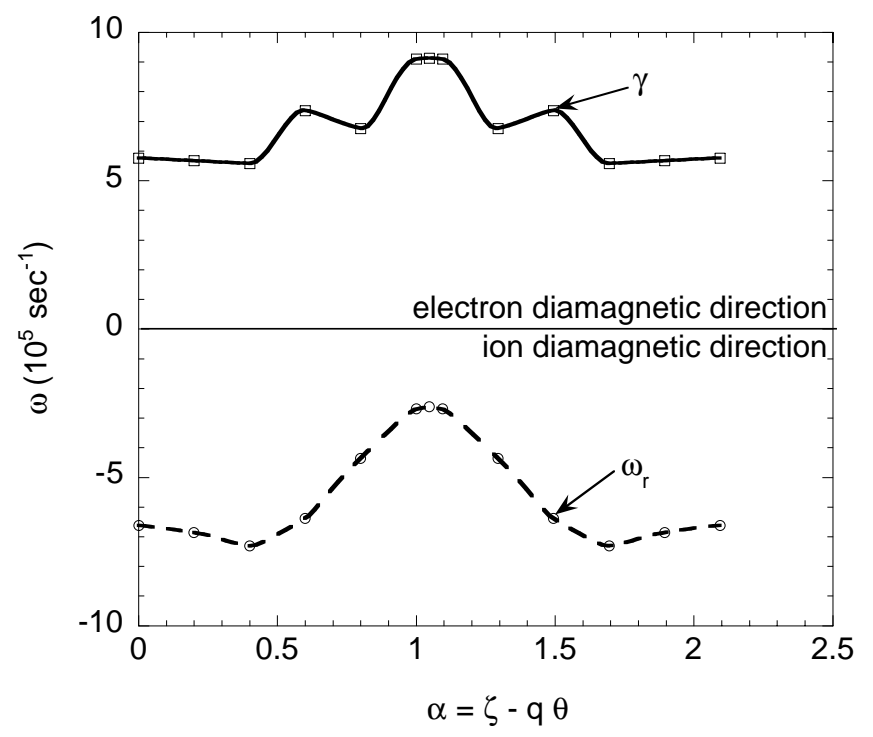

FIG. 9. Variation of $\gamma$ and $\omega_{r}$ with magnetic field line label $\alpha \equiv \zeta-q \theta$, for ITG root with $\eta_{i}=\eta_{e}=3.0$ and $n=25$, for case of Figs. 2 and 5. 
seen that the growth rate changes very little with $T_{\epsilon} / T_{i}$ when $T_{i}$ is varied, but increases substantially with $T_{e} / T_{i}$ when $T_{e}$ is varied. Both this behaviour and the opposite variation with $T_{e}$ have been seen in tokamak cases, depending on the regime considered.

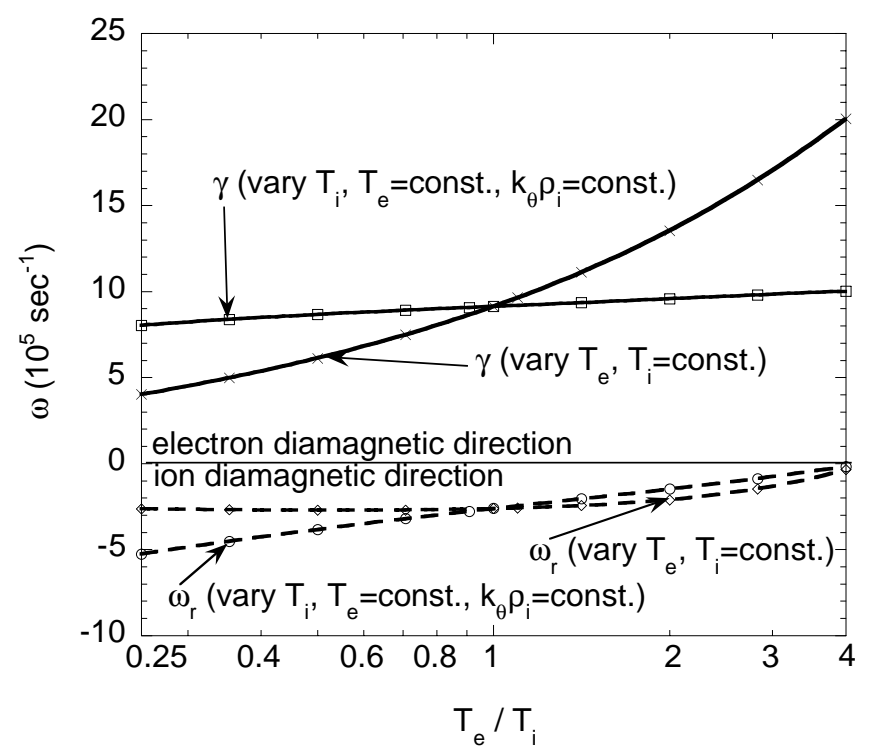

FIG. 10. Variation of $\gamma$ and $\omega_{r}$ with $T_{e} / T_{i}$, for ITG root with $\eta_{i}=\eta_{e}=3.0$ and $k_{\theta} \rho_{i}(\theta=0)=0.3983$, for case of Figs. 2 and 5 .

Finally, we vary the chosen magnetic surface (minor radius), and follow the variation of the ITG root for fixed $\eta_{i}=\eta_{e}=3.0$ and fixed $k_{\theta} \rho_{i}(\theta=0)=0.3983$, with $n$ varying so as to maintain that value, and with the other parameters having their standard values. The growth rate, shown in Fig. 11, peaks radially around $s \simeq 0.8$, but the ITG root is unstable for all radii examined from $s=0.4$ to $s=0.95$. The real frequency, also shown in Fig. 11 , is in the ion diamagnetic direction for all of these radii, but rises to make a transition to the electron diamagnetic direction very near the plasma boundary.

\section{COLLISIONAL EFFECTS}

In the original axisymmetric version of the FULL code, ${ }^{4,5,14}$ collisions were modeled by means of a "Krook" operator. The physical process involved there was a two-step effective 


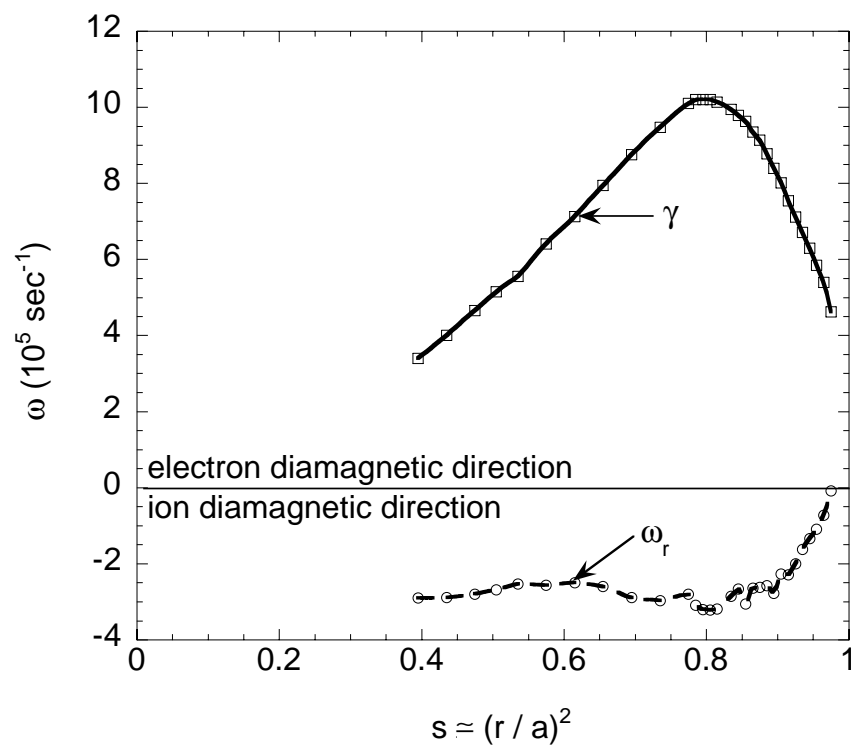

FIG. 11. Variation of $\gamma$ and $\omega_{r}$ with $s$, for ITG root with $\eta_{i}=\eta_{e}=3.0$ and $k_{\theta} \rho_{i}(\theta=0)=0.3983$, for case of Figs. 2 and 5 .

decorrelation of the particle's parallel motion by means of a pitch-angle scattering from the toroidally trapped range of pitch angles to the untrapped range, and then another scattering back from the untrapped range to the toroidally trapped range. The net effect of this two-step process was to give an enhancement of the effective collision frequency, over the normal collision frequency, that increased as the particle's pitch angle became closer to the trapped-untrapped boundary. In fact, good agreement with the results of a comparable calculation using a Lorentz collision operator was obtained ${ }^{14}$ when a divergence of proper strength at the trapped-untrapped boundary was built into the effective collision frequency. The axisymmetric Krook collision operator is given in detail in Ref. 14, and has an effective electron collision frequency

$$
\nu_{f e}^{\mathrm{as}}\left(E, \Lambda, \epsilon_{0}, h_{\min }\right)=\frac{\nu_{e}(E) \epsilon_{0}}{\left|h_{\min }-\Lambda\right|^{2}} \frac{0.111 \delta\left(\epsilon_{0}\right)+1.31}{11.79 \delta\left(\epsilon_{0}\right)+1},
$$

and with the ions either being treated with a similar collision operator or treated as collisionless. Here, the pitch angle variable $\Lambda \equiv \mu B_{0} / E, E$ and $\mu$ are the particle energy and magnetic moment, $B_{0}$ is the $\theta$ average of $B(\theta), \delta\left(\epsilon_{0}\right)=\left[|\omega| /\left(\nu_{e i} Z_{f} \times 37.2 / \epsilon_{0}\right)\right]^{1 / 3}$, $Z_{f} \equiv \sum_{i} Z_{i}^{2} n_{i} / n_{e}, \nu_{e i} \equiv 4 \pi n_{e} e^{4} \ln \lambda /\left[\left(2 T_{e}\right)^{3 / 2} m_{e}^{1 / 2}\right], \nu_{e}(E) \equiv\left[\nu_{e i} /\left(v / v_{e}\right)^{3}\right]\left[Z_{f}+H_{e e}\left(v / v_{e}\right)\right]$, 
$v_{e} \equiv\left(2 T_{e} / m_{e}\right)^{1 / 2}$, and $H_{\epsilon e}(x) \equiv \exp \left(-x^{2}\right) /(\sqrt{\pi} x)+\left[1-1 /\left(2 x^{2}\right)\right] \operatorname{erf}(\mathrm{x})$. As a matter of notation, we write $B=B_{0} / h(\theta)$, so that $h(\theta)=B_{0} / B(\theta)$ and $h_{\min }$ and $h_{\max }$ are the minimum and maximum values of $h(\theta)$. In the simplest toroidal equilibrium with circular magnetic surfaces, $h(\theta)=1+\epsilon_{0} \cos (\theta)$, with $\epsilon_{0}=r / R$, so that $h_{\min }=1-\epsilon_{0}$ and $h_{\max }=1+\epsilon_{0}$. With these choices, good agreement was obtained for the results for the linear growth rate with the results of a comparable calculation using a Lorentz collision operator, over the entire applicable range of collision frequencies. ${ }^{14}$ For the numerically-calculated axisymmetric MHD equilibria that we now generally employ, $h(\theta)$ comes from the MHD equilibrium calculation, $h_{\min }$ and $h_{\max }$ are the (numerically-determined) minimum and maximum of $h(\theta)$, respectively (corresponding to the maximum and minimum of $B(\theta)$, respectively), and $\epsilon_{0} \equiv\left(h_{\max }-h_{\min }\right) / 2$.

In the present nonaxisymmetric geometry, we can have multiple classes of helicallytrapped particles, in addition to the classes of toroidally-trapped and untrapped particles that are present in axisymmetric geometry. The two-step enhancement process for the effective collision frequency can also occur for transitions from one trapped-particle class to another, along with the inverse transitions, and can be modeled by a divergence in the effective collision frequency at the pitch-angle boundary between the two trapped-particle classes. All of the local minima of $h(\theta)$ (maxima of $B(\theta)$ ) are found numerically in the extended FULL code, as well as the maximum and minimum of $h(\theta)$ over the entire range of $\theta$, denoted as $h_{\max }$ and $h_{\min }$, respectively. The local minima which should be most important for this process for a given trapped particle are the two local minima just outside the turning points $\theta_{1}$ and $\theta_{2}$ (with $\theta_{1}<\theta_{2}$ ) for that trapped particle. Labeling the local minimum of $h(\theta)$ just to the left of $\theta_{1}$ as $h_{\mathrm{min}-1}$, and the local minimum of $h(\theta)$ just to the right of $\theta_{2}$ as $h_{\min -\mathrm{r}}$, we define $\epsilon_{01} \equiv\left(h_{\max }-h_{\min -1}\right) / 2$, and $\epsilon_{0 \mathrm{r}} \equiv\left(h_{\max }-h_{\min -\mathrm{r}}\right) / 2$. These sorts of extrema and turning points are illustrated in Fig. 12. In terms of the axisymmetric effective collision frequency $\nu_{f e}^{\mathrm{as}}\left(E, \Lambda, \epsilon_{0}, h_{\mathrm{min}}\right)$ defined in Eq. 5, the new nonaxisymmetric effective collision frequency used is given by 


$$
\nu_{f e}^{\mathrm{nas}}(E, \Lambda)=\frac{1}{2}\left(\nu_{f e}^{\mathrm{as}}\left(E, \Lambda, \epsilon_{01}, h_{\mathrm{min}-1}\right)+\nu_{f e}^{\mathrm{as}}\left(E, \Lambda, \epsilon_{0 \mathrm{r}}, h_{\mathrm{min}-\mathrm{r}}\right)\right)
$$

for each separate class of trapped particles. For convenience, we define the usual tokamak dimensionless collisionality parameter $\nu_{e}^{*} \equiv \nu_{e i} Z_{f} /\left(\epsilon_{0}^{3 / 2} v_{e} / L_{c}\right) \propto n_{e}$, where $\epsilon_{0} \equiv\left(h_{\max }-\right.$ $\left.h_{\min }\right) / 2$ and $L_{c} \equiv \int d \theta\left[\sqrt{g} B / \Psi^{\prime}(s)\right] / \int d \theta$, which reduces to $q R$ in the axisymmetric, circular cross-section limit.

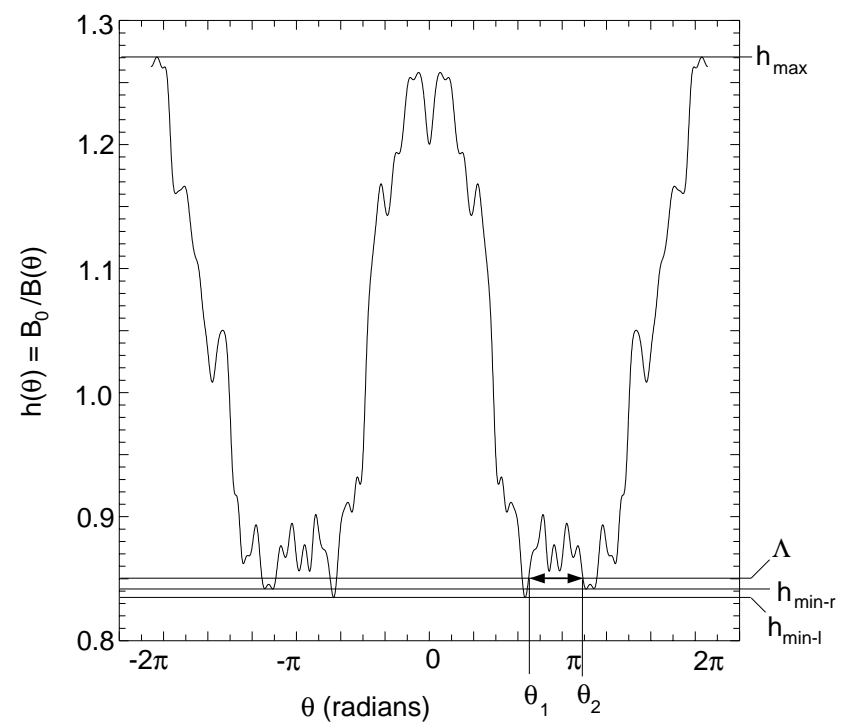

FIG. 12. Variation of $h(\theta) \equiv B_{0} / B(\theta)$ along chosen field line for case of Fig. 2, showing maximum $h_{\max }$ and minima $h_{\min -1}$ and $h_{\min -\mathrm{r}}$, particle turning points $\theta_{1}$ and $\theta_{2}$, and pitch angle variable $\Lambda$.

This effective collision frequency is a straightforward and usable generalization of the axisymmetric collision frequency, for the situation with multiple classes of trapped particles. and reduces to it exactly in the limit of axisymmetric geometry, where the only minima of $h(\theta)$ are those that divide the toroidally trapped particles from the untrapped particles (and all the minima are equal). This model collision operator, as given in Ref. 14, conserves particle number and energy for each species, but not momentum. It should be acceptably accurate in the banana regime $\left(\nu_{e}^{*}<1\right)$ and at the lower end of the plateau regime $\left(\nu_{e}^{*} \gtrsim 1\right)$.

The effects of increasing collisionality on $\gamma$ and $\omega_{r}$ are shown in Fig. 13, for the cases considered in Sec. III B, versus $n_{e}$ or $\nu_{e}^{*} \propto n_{e}$. Results are shown for the TEM root with 
$\eta_{i}=\eta_{e}=0.0$ and $n=29\left(k_{\perp} \rho_{i}(\theta=0)=0.4620\right)$ and for the ITG root with $\eta_{i}=$ $\eta_{e}=3.0$ and $n=25\left(k_{\perp} \rho_{i}(\theta=0)=0.3983\right)$. For these results, ion-ion collisions are included with an effective collision frequency of the same general form as Eq. 6, but ionion collisions have a very small effect for the normal range of parameters. One of the major effects of the increasing effective collision frequency is to reduce the trapped-electron contribution to the eigenmode integral equation, because of the presence of the effective collison frequency in the resonant denominator. ${ }^{4,5}$ The growth rate in Fig. 13(a) for the TEM root decreases almost to zero as $n_{e}$ or $\nu_{e}^{*}$ increases, because the trapped electrons provide almost all of the destabilization for this root through the trapped-electron time-average magnetic drift precession resonance mechanism. The growth rate for the ITG root decreases less, because most of the destabilization for it comes from the nonresonant ion temperature gradient mechanism. Another effect of the trapped-electron contribution to the eigenmode integral equation is to push the real frequency $\omega_{r}$ towards the ion diamagnetic direction. The reduction of the trapped-electron contribution with increasing $n_{e}$ or $\nu_{e}^{*}$ allows $\omega_{r}$ to move back in or towards the electron diamagnetic direction for both the TEM root and the ITG root, as seen in Fig. 13(b).

The results of varying $\eta_{i}=\eta_{e}$ at fixed density $n_{e}=2.828 \times 10^{20} \mathrm{~m}^{-3}$ or $\nu_{e}^{*}=0.7665$ are shown in Fig. 6, along with the collisionless results. Collisions at this level give partial stabilization and a shift in the critical values of $\eta_{i}=\eta_{e}$ for both the TEM root and the ITG root, as seen in Fig. 6(a). The real frequencies for both roots are also shifted towards or further into the elecron diamagnetic direction, as seen in Fig. 6(b).

\section{CONCLUSIONS}

A well-established comprehensive kinetic code for analyzing axisymmetric tokamak linear microinstabilities has now been generalized to deal with nonaxisymmetric stellarator geometry. This new version of the FULL code has all of the same kinetic dynamics as the axisymmetric version, and is currently operational in the electrostatic limit. A model 

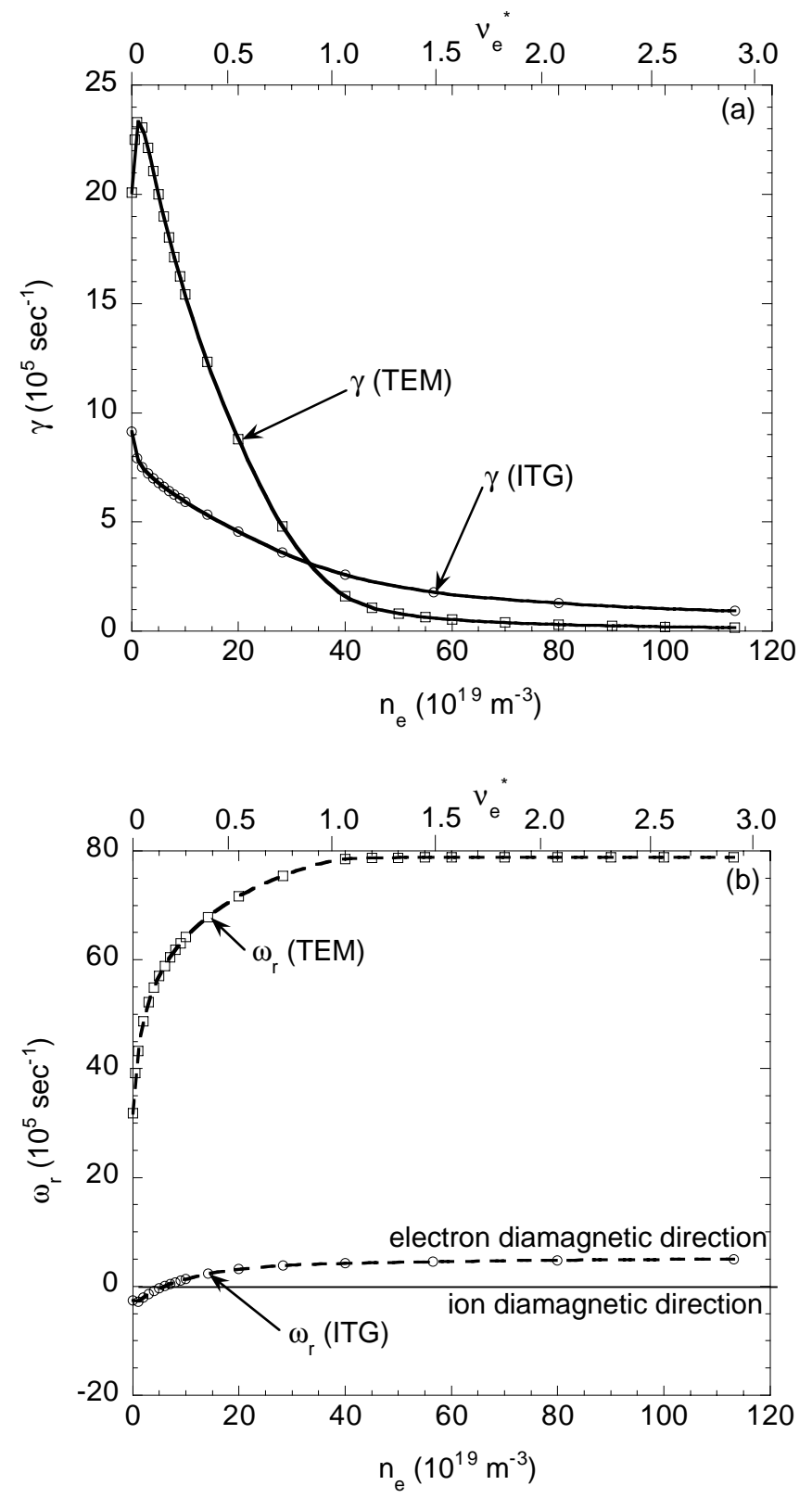

FIG. 13. Variation of (a) growth rate $\gamma$ and (b) real frequency $\omega_{r}$ with electron density $n_{e}$ and collisionality parameter $\nu_{e}^{*}$, for case of Figs. 2 and 5 , with $\eta_{i}=\eta_{e}=3.0$ and $n=25$ $\left(k_{\theta} \rho_{i}(\theta=0)=0.3983\right)$ for ITG root, and with $\eta_{i}=\eta_{e}=0.0$ and $n=29\left(k_{\theta} \rho_{i}(\theta=0)=0.4620\right)$ for TEM root. 
collision operator, which has been successfully benchmarked against more rigorous collision models, was previously used in the axisymmetric version of the code, and has now been extended to accommodate scenarios where there are multiple classes of trapped particles, i.e., both one class of toroidally trapped and multiple classes of helically trapped particles. The needed MHD equilibrium is calculated using the three-dimensional VMEC equilibrium code, with the results being converted to Boozer coordinates by the TERPSICORE code, and the requisite input quantities along a chosen magnetic field line being constructed by the VVBAL code. Good agreement was obtained between the axisymmetric and nonaxisymmetric versions of the FULL code using "matched" ARIES-RS axisymmetric MHD equilibria computed both by the two-dimensional JSOLVER code and by the three-dimensional VMEC code.

Results have been obtained for the toroidal drift mode for an MHD equilibrium generated in the course of the ongoing design studies for the NCSX stellarator. Two roots are found to be unstable for the parameters examined. One corresponds to the ion-temperature-gradient (ITG) mode, and the other to the trapped-electron mode (TEM). The changes in the linear growth rates and real frequencies were examined as $\eta_{i}=\eta_{e}, n\left(\right.$ or $\left.k_{\perp} \rho_{i}(\theta=0) \propto n\right)$, $\theta_{0}, \alpha, T_{e} / T_{i}, s \simeq$ average $(\mathrm{r} / \mathrm{a})^{2}$, and $n_{e}\left(\right.$ or $\left.\nu_{e}^{*} \propto n_{e}\right)$ are varied. Overall, the results of this variation are generally similar to what is observed in normal tokamak cases. This is not surprising, since this particular quasiaxisymmetric stellarator equilibrium is designed to have some of the same properties as an axisymmetric tokamak equilibrium, but only in Boozer coordinates. For other stellarator cases, that will be investigated in the future, the magnetic geometry in Boozer coordinates can be very different from that for a tokamak, and the microinstability results can be correspondingly different. For instance, in an experiment such as LHD, ${ }^{1}$ the influence of the helically-trapped particles is expected to be very much larger than for a quasiaxisymmetric stellarator.

In the future, a further extension of the present version of the nonaxisymmetric FULL code is planned, in order to make it fully electromagnetic. The present electrostatic version will be reasonably accurate for toroidal drift modes for low to moderate values of $\beta$, where the 
dominant finite- $\beta$ effects enter through the MHD equilibrium, for instance as magnetic axis shifts (Shafranov shifts), as changes in magnetic surface shape, etc. However, at higher values

of $\beta$, the direct electromagnetic coupling of $\hat{\phi}$ to $\hat{A}_{\|}$and $\hat{A}_{\perp}$ will also become important. Another possible extension is the implementation of a rotation model, of the type that has already been implemented in the axisymmetric version of the FULL code. ${ }^{18}$

It should be noted that the present calculation is only a lowest-order, radially-local calculation in the ballooning hierarchy. The very simplest method ${ }^{19,20}$ of going to higher order, to obtain a radially-global eigenfrequency result, is to use the so-called $1 / n$ correction term, just as in axisymmetric kinetic cases, ${ }^{21}$ with $n$ no longer being restricted to integer values. To go beyond this, to more general WKB-type formulations, is at present an active area of research, ${ }^{22-24}$ even for the simpler dynamics of ideal MHD ballooning modes.

The nonaxisymmetric generalization of the FULL code developed in the present studies can contribute valuable information to the design process for new stellarators. Specifically, identification of magnetic geometries and equilibrium profile shapes with reduced microinstability growth rates would be suggestive of configurations with improved anomalous transport properties. The present capability can also be employed in the analysis of experimental discharges, using experimentally reconstructed MHD equilibria and experimentally-measured equilibrium profile shapes, to assess whether the theoretically-predicted microinstabilities can be responsible for experimentally observed fluctuations and associated anomalous transport. Motivated by these considerations, future studies will be made even more realistic, by including impurities and hot beam ions, along with the electromagnetic (finite- $\beta$ ) dynamics.

\section{ACKNOWLEDGMENTS}

The authors would like to thank Dr. C. Kessel for providing the JSOLVER ARIES-RS MHD equilibrium, and Prof. R. L. Dewar for useful discussions. This work was supported by United States Department of Energy Contract No. DE-AC02-76-CHO-3073. 


\section{REFERENCES}

${ }^{1}$ M. Fujiwara, H. Yamada, A. Ejiri, et al., Plasma Physics and Controlled Nuclear Fusion Research, 1998 (International Atomic Energy Agency, Vienna, 1999), paper IAEA-CN$69 / \mathrm{EX} 2 / 3$.

${ }^{2}$ F. Wagner, Plasma Physics and Controlled Nuclear Fusion Research, 1998 (International Atomic Energy Agency, Vienna, 1999), paper IAEA-CN-69/OV2/4.

${ }^{3}$ A. Reiman, L. Ku, D. Monticello, et al., Plasma Physics and Controlled Nuclear Fusion Research, 1998 (International Atomic Energy Agency, Vienna, 1999), paper IAEA-CN$69 / \mathrm{ICP} / 06$.

${ }^{4}$ G. Rewoldt, W. M. Tang, and M. S. Chance, Phys. Fluids 25, 480 (1982).

${ }^{5}$ G. Rewoldt, W. M. Tang, and R. J. Hastie, Phys. Fluids 30, 807 (1987).

${ }^{6}$ S. P. Hirshman and D. K. Lee, Comput. Phys. Commun. 39, 161 (1986).

${ }^{7}$ S. P. Hirshman, U. Schwenn, and J. Nuehrenberg, J. Comput. Phys. 87, 396 (1990).

${ }^{8}$ A. H. Boozer, Phys. Fluids 24, 904 (1980).

${ }^{9}$ D. V. Anderson, W. A. Cooper, R. Gruber, S. Merazzi, and U. Schwenn, Int. J. Supercomp. Appl. 4, 34 (1990).

${ }^{10}$ W. Anthony Cooper, Plasma Phys. and Controlled Fusion 34, 1011 (1992).

${ }^{11}$ S. C. Jardin, C. E. Kessel, C. G. Bathke, D. A. Ehst, T. K. Mau, F. Najmabadi, T. W. Petrie and the ARIES Team, Fusion Engr. and Design 38, 27 (1997).

${ }^{12}$ J. DeLucia, S. C. Jardin, and A. M. M. Todd, J. Comput. Phys. 37, 183 (1980).

${ }^{13}$ J. L. Johnson, H.E. Dahled, J. M. Greene, R. C. Grimm, Y. Y. Hsieh, S. C. Jardin, J. Manickam, M. Okabayashi, R. G. Storer, A. M. M. Todd, D. E. Voss, and K. E. Weimer, Journal of Comput. Phys. 32, 212 (1979). 
${ }^{14}$ M. Kotschenreuther, G. Rewoldt, and W. M. Tang, Comp. Phys. Comm. 88, 128 (1995).

${ }^{15}$ M. A. Beer, G. W. Hammett, G. Rewoldt, E. J. Synakowski, M.C. Zarnstorff, and W. Dorland, Phys. Plasmas 4, 1792 (1997).

${ }^{16}$ A. Reiman, B. Blackwell, A. Boozer, et al., in Proceedings of the 26th European Physical Society Conference on Controlled Fusion and Plasma Physics Research, Maastricht, the Netherlands, June 14-18, 1999, (European Physical Society, Petit-Lancy, 1999), paper TL18.

${ }^{17}$ G. Rewoldt and W. M. Tang, Phys. Fluids B 2, 318 (1990).

${ }^{18}$ G. Rewoldt, M. A. Beer, M. S. Chance, T. S. Hahm, Z. Lin, and W. M. Tang, Phys. Plasmas 5, 1815 (1998).

${ }^{19}$ R. L. Dewar and A. H. Glasser, Phys. Fluids 26, 3038 (1983).

${ }^{20}$ R. L. Dewar, private communication (1998).

${ }^{21}$ E. A. Frieman, G. Rewoldt, W. M. Tang, and A. H. Glasser, Phys. Fluids 23, 1750 (1980).

${ }^{22}$ W. A. Cooper, D. B. Singleton, and R. L. Dewar, Phys. Plasmas 3, 275 (1996).

${ }^{23}$ R. L. Dewar, P. Cuthbert, J. L. V. Lewandowski, H. J. Gardner, D. B. Singleton, M. Persson, and W. A. Cooper, J. Korean Phys. Soc. (Proc. Suppl.) 31, S115 (1997).

${ }^{24}$ P. Cuthbert, J. L. V. Lewandowski, H. J. Gardner, M. Persson, D. B. Singleton, R. L. Dewar, N. Nakajima, and W. A. Cooper, Phys. Plasmas 5, 2921 (1998). 